\title{
Non-isothermal flux penetration in type II superconductors
}

\author{
E.A. Gijsbertse, L.J.M. van de Klundert, M.L.D. van Rij and W.J. van Weezep
}

The properties of hard superconductors are closely described by the critical state model and its extensions, which allow for the finite nature of the first critical field $B_{\mathrm{c} 1}$ and flux flow or flux creep effects. For the technical application of hard superconductors, however, the stability of the critical state in both ac and dc circuits is of essential importance. These instabilities, attended by a large increase of internal flux (flux jump), and their prevention have been studied parallel to the development of hard superconductors. These studies can be classified into three major categories.

Firstly, criteria have been derived predicting the external field value above which instabilities may occ1.. These criteria have the form:

$$
\left.B_{\mathrm{fj}}=\Delta\left(\mu_{0} C j_{\mathrm{c}} /-\partial j_{\mathrm{c}} / \partial T\right)\right)^{1 / 2}
$$

in which $j_{\mathrm{c}}$ is the field and temperature dependent critical current density, $C$ the specific heat per unit volume and $\Delta$ a constant depending on geometry and on the physical assumptions. In the derivation of these criteria it is assumed that the heat transport inside the sample and from the sample to the environment can be neglected. Therefore they are called locally adiabatic criteria. For the constant $\Delta$, Hancox ${ }^{1}$ finds $\Delta=\sqrt{ } 2$, Swartz and Bean ${ }^{2}$ and Wipf ${ }^{3}$ $\Delta=\pi / 2$ and Melville ${ }^{4}$ and Takeo et al. ${ }^{5} \Delta=\sqrt{3}$. In fact these criteria apply only for high $\kappa$ superconductors. For low $\kappa$, type II superconductors like niobium, the final value of $B_{c 1}$ and the superheating of the Meissner state ${ }^{6,7}$ have to be considered. Also the presence of a surface barrier, describing surface pinning and metastable states can largely influence the jump field $B_{\mathrm{fj}}$ in practical superconductors as was pointed out by $\mathrm{Kim}$ et al. ${ }^{8}$ All these theoretical treatments predict instability against local perturbation in the steady state.

It was observed that the sweep rate of the applied magnetic field (triangular or sinusoidal waveform) affects the value of the jump field. ${ }^{9}$ In order to derive sweep rate dependent flux jump criteria, it is necessary to introduce voltage-current relations for over-critical currents describing the flux creep ${ }^{10}$ or the flux flow state. ${ }^{11,12}$ These criteria have been obtained under various assumptions about heat conduction inside the sample and from the sample to the environment. It has been found that the

The authors are at Twente University of Technology, Department of Applied Physics, POB 217, 7500 AE Enschede, The Netherlands. Paper received 12 June 1979. reduction of the jump field due to the sweep rate is linear on a semi-logarithmic plot for small $\mathrm{d} B_{\mathrm{a}} / \mathrm{d} t$ values $^{3}$ or on a log-log plot in the flux creep state.

As has already been pointed out by Takeo et al. ${ }^{5}$, the fundamental understanding of the mechanism of flux jumps, especially under ac conditions, is lacking. Also the agreement between theoretical and experimental results is of a qualitative nature, mostly due to the simplifying assumptions necessary in the mathematical treatment. A third approach therefore is the numerical solution of the fundamental equations governing the flux jump behaviour. To our knowledge only a few studies on this subject have been published, ${ }^{13,14}$ none of them cover the dependence of the flux jump field on the sweep rate of the externally applied field, and the results available are only valid for virgin samples. The equations to be solved are (in slab geometry) the heat transport equation:

$$
C \frac{\partial T}{\partial t}=\frac{\partial}{\partial x}\left(K \frac{\partial T}{\partial x}\right)+P(x, t)
$$

in which $K$ is the temperature dependent thermal conductivity throughout the sample, and the flux diffusion equation:

$$
\frac{1}{\mu_{0}} B \frac{\partial B}{\partial x}=F_{\mathrm{p}}(x, T)+\eta(T) \int_{0}^{x} \frac{\partial B}{\partial t} \mathrm{~d} x^{\prime}
$$

This equation is found from the well accepted relation:

$$
j=j_{\mathrm{c}}+\sigma_{\mathrm{ff} \cdot} E
$$

making use of Maxwell's equations and inserting:

$$
j_{\mathrm{c}}=F_{\mathrm{p}}(x, T) / B
$$

for the critical current density $\left(F_{\mathrm{p}}\right.$ denotes the pinning force). The flux flow conductivity $\sigma_{\mathrm{ff}}$ is replaced by:

$$
\sigma_{\mathrm{ff}}=\sigma_{\mathrm{n}} \cdot B_{\mathrm{c} 2}(T) / B=\eta(T) / B
$$

In these equations only variations of $B$ and $T$ perpendicular to the surface of the superconducting slab are taken into account. Also thermo-magnetic and galvano-magnetic effects are neglected. The two diffusion equations, (2) and (3), are coupled by the dissipation which can be written as:

$$
P(x, t)=j . E=\frac{1}{\mu_{0}} \frac{\partial B}{\partial x} \int_{0}^{x} \frac{\partial B}{\partial t} \mathrm{~d} x^{\prime}
$$


The boundary conditions for the temperature equation are:

$$
\begin{aligned}
& \left.\frac{\partial T}{\partial x}\right|_{x=0}=0 \\
& \left.\frac{\partial T}{\partial x}\right|_{x=x_{\mathrm{t}}} \text { is continuous; and } \\
& \left.K \frac{\partial T}{\partial x}\right|_{x=\mathrm{d}}=-Q_{0}\left[T(\mathrm{~d})-T_{0}\right]^{\mathrm{n}}
\end{aligned}
$$

Here $x_{t}$ indicates the position to which the flux variation and with it the dissipation have penetrated inside the slab; $Q_{0}$ determines the heat transfer from the sample to the surrounding liquid helium and $n$ ranges from 1 to 3 in practical cases. $T_{0}$ is the temperature of the surrounding helium. Various attempts have been made to solve these equations ${ }^{13,14}$ using different assumptions about flux pinning and flux flow, but so-far no satisfying solution has been obtained.

In the present work measurements on flux jumps are presented together with numerical calculations. A niobium single crystal slab (dimensions: $16 \times 4 \times 0.5 \mathrm{~mm}^{3}$ ) is placed in a triangular or trapezoidal ${ }^{15}$ ac field of maximum amplitude of $0.5 \mathrm{~T}$. The voltage induced in a pick-up coil is registered carefully using a transient recorder. The sweep rate of the external field was varied from $10^{-3}$ to $10 \mathrm{Ts}^{-1}$. The experimental results are presented in the next section. A simplified model, which accounts for surface pinning only, is introduced and solved numerically. This model explains part of the observed phenomena. The introduction of this new model was suggested by the experimental hysteresis loops in which very large surface currents, already occurring at the second critical field $B_{\mathrm{c} 2}$, are observed. The model, however, may also be useful to explain instabilities in thin layers of $\mathrm{Nb}_{3} \mathrm{Sn}$ or $\mathrm{Nb}_{3} \mathrm{Ge}$ produced by means of diffusion of $\mathrm{Sn}$ and $\mathrm{Ge}$ in $\mathrm{Nb}$-slabs or films. ${ }^{16}$ The major part of the measurements were done at $4.2 \mathrm{~K}$ in liquid helium. At lower temperatures a different behaviour was observed, but this has not yet been investigated systematically. However, these differences do not violate the fundamental concepts of our model.

\section{Experimental results}

The ac response of a niobium single crystal deviates sharply from that of a niobium polycrystalline slab of approximately the same size. Fig. 1 shows some plots of the average induction $B_{\mathrm{av}}$ inside the sample versus the externally applied induction $B_{\mathrm{a}}$ for both samples. In the $B_{\mathrm{av}}$ vs $B_{\mathrm{a}}$ loops of the single crystal the flux jumps can be seen clearly. The behaviour of the polycrystalline sample can be understood qualitatively in terms of the isothermal critical state model allowing for small surface barriers and flux flow. ${ }^{15}$ In the case of the single crystal where flux jumps arise, the isothermal description is no longer valid. Experiments have been performed in triangular and trapezoidal ac fields in a wide range of $\mathrm{d} B_{\mathrm{a}} / \mathrm{d} t$ values. The equipment used for the measurements will be described elsewhere. ${ }^{17}$ Two-pick-up coils are used to record the induced voltage: a small one of 200 windings to measure the voltage peak and a large one (2000 turns) for the rest of the signal. Both pick-up coils are placed in such a way that good thermal contact between sample surface and the liquid helium bath is assured.
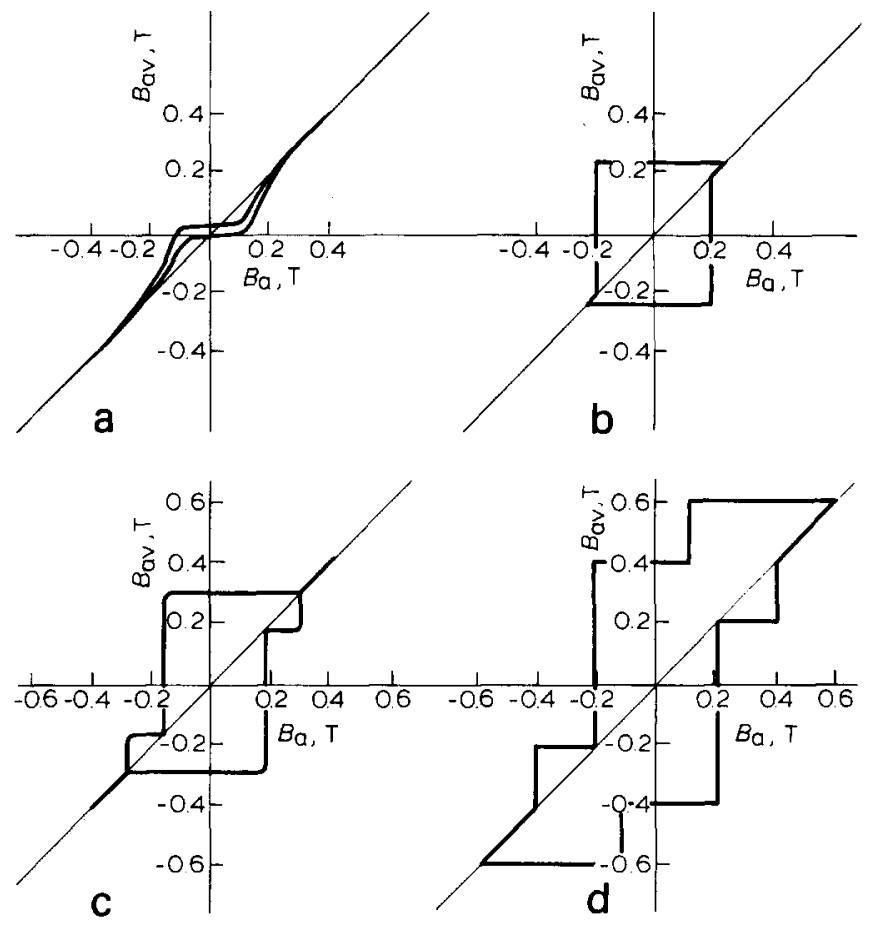

Fig. 1 Hysteresis loops for a polycrystalline niobium slab (a) and a niobium single crystal $(\mathrm{b}-\mathrm{d}) . \mathrm{a}-b_{0}=400 \mathrm{mT} ; \mathrm{d} B_{\mathrm{a}} / \mathrm{d} t=3 \mathrm{Ts}^{-1}$; $T=4.2 \mathrm{~K} ; \mathrm{b}-b_{0}=230 \mathrm{mT} ; \mathrm{d} B_{\mathrm{a}} / \mathrm{d} t=5 \mathrm{Ts}^{-1} ; T=4.2 \mathrm{~K}$;

$c-b_{0}=500 \mathrm{mT} ; \mathrm{d} B_{\mathrm{a}} / \mathrm{d} t=3 \mathrm{Ts}^{-1} ; T=4.2 \mathrm{~K} ; \mathrm{d}-b_{0}=660 \mathrm{mT}$; $\mathrm{d} B_{\mathrm{a}} / \mathrm{d} t=0.1 \mathrm{Ts}^{-1} ; T=1.5 \mathrm{~K}$

The reproducibility of the jump field as a function of frequency and amplitude of the applied triangular or trapezoidal ac field, shows that no accidental circumstances initiate the flux jump. This makes a systematic investigation possible. The voltage induced in a pick-up coil around the sample has been measured systematically as a function of the sweep rate $\mathrm{d} B_{\mathrm{a}} / \mathrm{d} t$ and the amplitude $b_{0}$ of the ac field as well as the duration $\Delta t$ of the time intervals during which $\mathrm{d} B_{\mathrm{a}} / \mathrm{d} t=0$ (trapezoidal fields). Some results at lower temperatures have also been obtained for comparison. Fig. 2 shows the signal of the pickup coil, after compensation for the response of the empty part of the coil, as a function of applied field. The position of the jump, which itself cannot be seen on this scale, is indicated by an arrow.

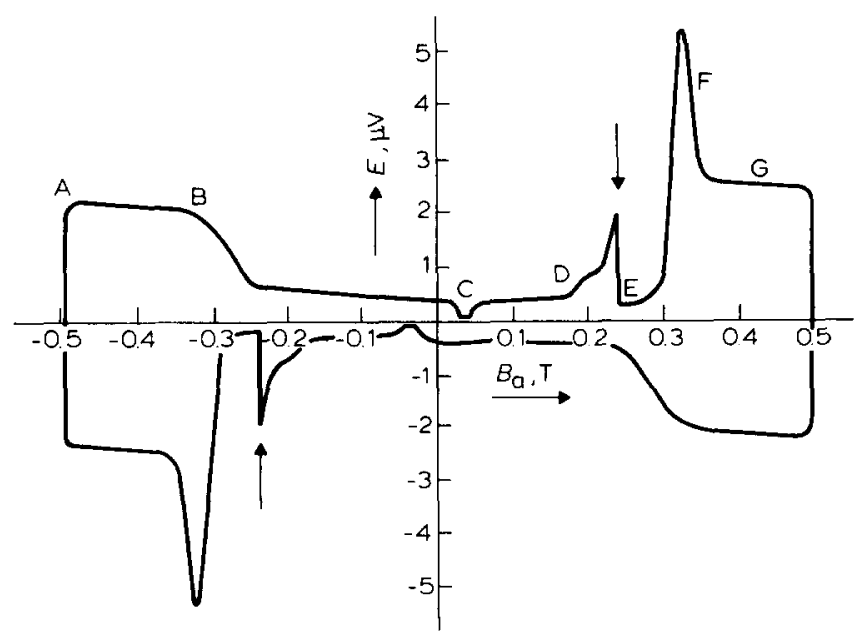

Fig. 2 Voltage induced in one turn around the single crystal versus applied field $\left(b_{0}=500 \mathrm{mT} ; \mathrm{d} B_{a} / \mathrm{d} t=0.1 \mathrm{Ts}^{-1}\right)$. The arrow indicates the position of the flux jump $\left(B_{\mathrm{f}]}=227 \mathrm{mT}\right)$; the letters $A-G$ are referred to in the text 
Apart from the region of the actual jump, a lot of other interesting features can be seen in this signal. From $A$ to $B$, the response of a normal metal in a linear increasing field is observed. ${ }^{18}$ At $B$, the second critical field, the penetration of flux is largely reduced. It is assumed that a surface screening current builds up and that the internal flux density is almost unchanged. Just after the external field has passed through zero, $\mathrm{C}$, a second reduction of the signal, almost to zero, is observed. At D, an increase of the flux penetration starts which finally results in a flux jump (arrow). Immediately after the jump the signal is again at the lowest level observed before E. Finally, it increases to a peak at $B_{\mathrm{c} 2}, \mathrm{~F}$, before entering the normal state. For the sake of clarity the return curve is shown. Two peculiar phenomena can be seen in this curve which to our knowledge have not been observed before. Firstly, complete flux screening starts at $B_{\mathrm{c} 2}$ and secondly, a strange dip at the zero passage of the external field is observed. These phenomena cannot be explained so far.

By integrating the observed signal, $B_{\mathrm{av}}$ vs $B_{\mathrm{a}}$ loops can be constructed. Three typical cases are shown in Fig. 1. For small amplitudes of the ac field $\left(b_{0}<B_{\mathrm{I}}\right)$, no flux penetration is observed. In the virgin state $B_{\mathrm{I}}$ is independent of frequency $\left(B_{\mathrm{I}}=220 \mathrm{mT}\right)$. If flux is alre wuy trapped in the sample $B_{\mathrm{I}}$ reduces with increasing $\mathrm{d} B_{\mathrm{a}} / \mathrm{d} t$. For larger amplitudes $\left(B_{\mathrm{I}}<b_{0}<B_{\mathrm{II}}\right)$, the hysteresis loop has the shape shown in Fig. 1b. The value of $B_{\text {II }}$ is about $320 \mathrm{mT}$ which suggests a relationship with $B_{\mathrm{c} 2}$. The value of the flux jump field $B_{\mathrm{fj}}$ can amount upto $280 \mathrm{mT}$ at very low $\mathrm{d} B_{\mathrm{a}} / \mathrm{d} t$ values. This value is larger than both the first critical field $B_{\mathrm{c} 1}$ and the thermodynamic critical field $B_{\mathrm{c}}$ so processes like superheating of the Meissner State cannot be responsible for the delay of flux exit and hence a surface pinning mechanism has to be assumed. By increasing the sweep rate, $B_{\mathrm{fj}}$ is reduced. Also by increasing the amplitude, $B_{\mathrm{fj}}$ can be reduced which suggests that $B_{\mathrm{fj}}$ can be reduced which suggests that $B_{\mathrm{fj}}$ depends on the amount of flux trapped in the sample as well. However, the total field screened by the surface current increases so both the external and internal induction affect the magnitude of the maximum screening current.

When the external field is increased above $B_{\mathrm{II}}$, the response of a normal metal is observed. ${ }^{18}$ In the vicinity of $B_{\mathrm{c} 2}$ this response can be obscured by the current carrying capacity of the surface layer between $B_{\mathrm{c} 2}$ and $B_{\mathrm{c} 3}$. When amplitudes larger than $B_{I I}$ are used, flux jumps occur independently of the amplitude at the same $B_{\mathrm{fj}}$ value, which is now only sweep rate dependent. At very large $\mathrm{d} B_{\mathrm{a}} / \mathrm{d} t$ values, the duration $\Delta t$ of the constant part of the trapezoidal ac field also affects the value of $B_{\mathrm{fj}}$. This can be understood from the fact that flux is already frozen in when the sample cools down from the previous flux jump. A variation of $\Delta t$ now implies a variation of the amount of flux which is frozen in.

In Fig. 4 the dependence of $B_{\mathrm{fj}}$ on $\mathrm{d} B_{\mathrm{a}} / \mathrm{d} t$ is given. The semilogarithmic dependence calculated by Wipf ${ }^{3}$ seems to apply, although no numerical confirmation can be obtained since Wipf did not give the value of the proportionality constant. The dependence of $B_{\mathrm{fj}}$ on amplitude is shown in Fig. 5 for some $\mathrm{d} B_{\mathrm{a}} / \mathrm{d} t$ values. A remarkable fact is the sudden change in $B_{\mathrm{fj}}$ when $b_{0}$ exceeds $B_{\mathrm{II}}$. For lower temperatures, the number of flux jumps can increase. For instance, at $T=1.5 \mathrm{~K}$ an incomplete jump occurs at $B_{\mathrm{a}}=-110 \mathrm{mT}$ in an increasing field and at $B_{\mathrm{a}}=210 \mathrm{mT}$ a complete jump is observed (see Fig. 1d). These $B_{\mathrm{fj}}$ values are independent of the sweep rate in the range $10^{-3}<$ $\mathrm{d} B_{\mathrm{a}} / \mathrm{d} t<10^{-1} \mathrm{Ts}^{-1}$. In Fig. 6a some experimental hysteresis loops at $4.2 \mathrm{~K}$ are shown for different $\mathrm{d} B_{\mathrm{a}} / \mathrm{d} t$ values. The amount of flux penetrating during the jump first increases with increasing $\mathrm{d} B_{\mathrm{a}} / \mathrm{d} t$, goes through a maximum and decreases at higher sweep rates. This can be understood from the rate of flux penetration just before the jump (see Fig. 6a). The corresponding voltage curves are given in Fig. $6 \mathrm{~b}$; the voltage peaks are shown in Fig. 3.

\section{Numerical considerations}

The set of equations (2-8) describing the thermal and magnetic behaviour of the sample can only be solved numerically. Even then a number of simplifying assumptions with respect to the dependence of the various material properties, like $C, K, \eta$ and $F_{\mathrm{p}}$, on temperature and magnetic induction have to be made. Also initial values of the flux and temperature distributions inside the sample and boundary conditions have to be imposed. The numerical solutions, found so $\mathrm{far}^{13}{ }^{14}$ do not apply to the experimental results we have reported, since in these calculations surface currents are not taken into account and only steady state solutions are obtained. Apart from this, the recovery of the superconducting state after a complete flux jump has to be considered. This recovery can only take place if the sample is allowed to cool down so non-adiabatic boundary conditions at the sample surface are inevitable.

During the last part of the voltage peak all peaks coincide (see Fig. 3). This part of the curve, where the sample is heated into the normal state, can be analysed with the help of a simple model. Physically, this situation is almost the same as the one in which a normal metal is suddenly

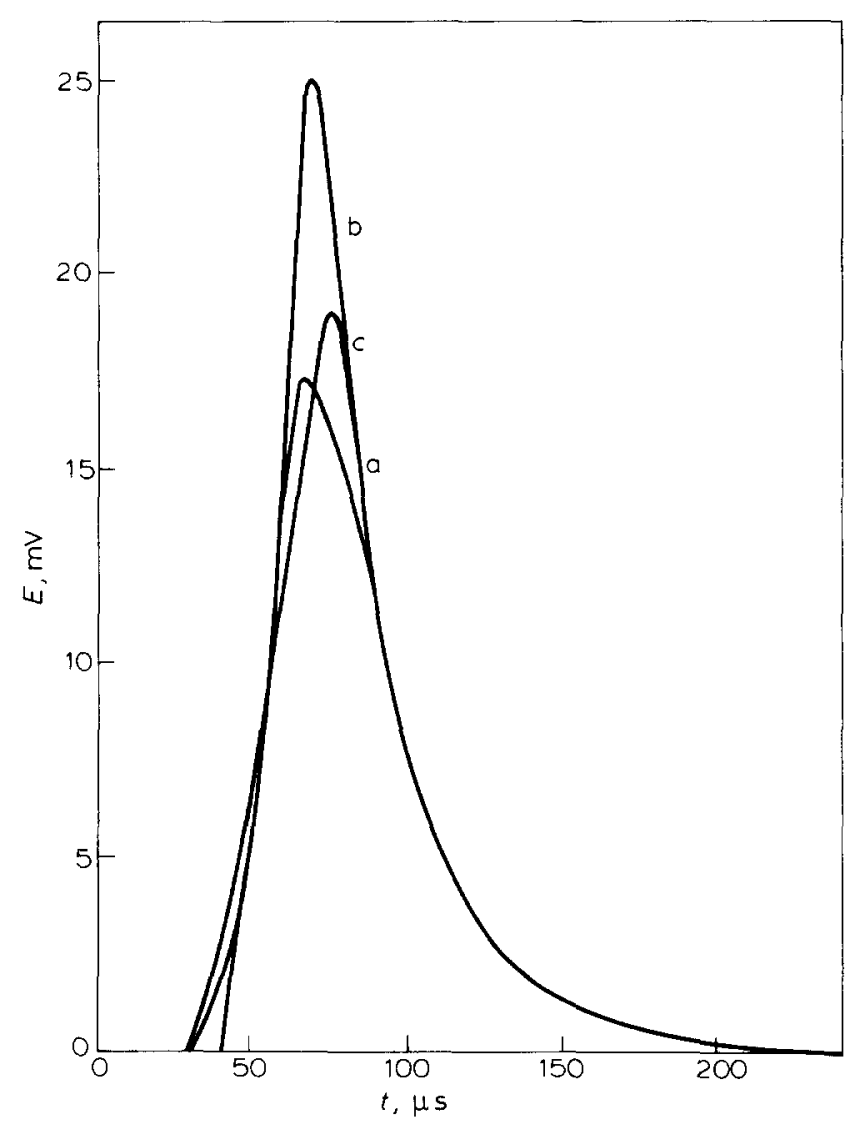

Fig. 3 Shape of the voltage peaks during the flux jump $\mathrm{a}-\mathrm{d} B_{\mathrm{a}} / \mathrm{d} t=2 \mathrm{mTs}^{-1} ; \mathrm{b}-\mathrm{d} B_{\mathrm{a}} / \mathrm{d} t=0.1 \mathrm{Ts}^{-1} ; \mathrm{c}-\mathrm{d} B_{\mathrm{a}} / \mathrm{dt}=3 \mathrm{Ts}^{-1}$ 


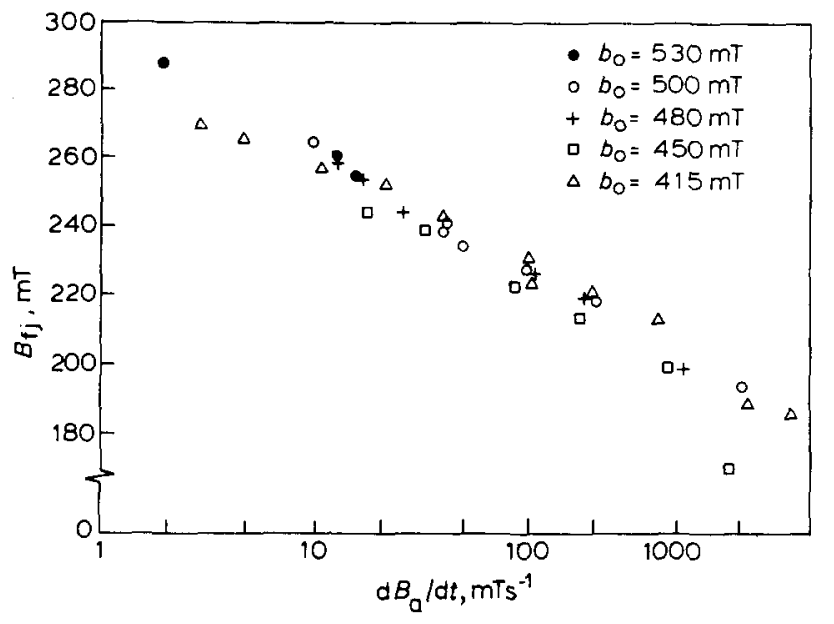

Fig. 4 The external field at which the flux jump takes place as a function of $d B_{a} / d t$

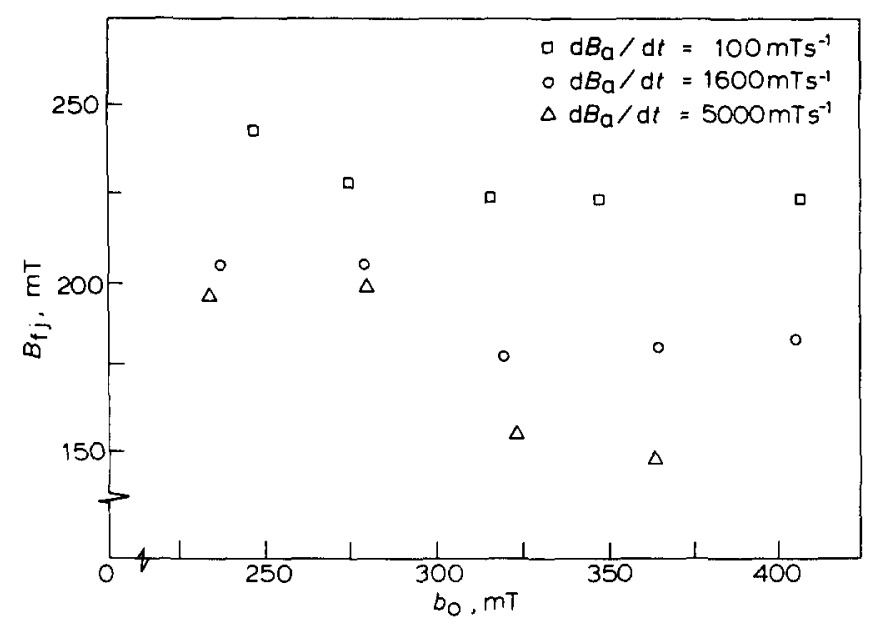

Fig. 5 The flux jump field $B_{\mathrm{fj}}$ vs amplitude of the ac field for various values of $\mathrm{d} B_{\mathrm{a}} / \mathrm{dt}$

placed in a constant magnetic field $B^{\prime}$. If the normal conductivity $\sigma_{\mathrm{n}}$ is temperature independent, only the magnetic diffusion equation has to be taken into account which in this case can be written as:

$$
\frac{\partial^{2} B}{\partial x^{2}}=\mu_{0} \sigma_{n} \frac{\partial B}{\partial t}
$$

The boundary conditions are:

$$
\begin{aligned}
& B(x, 0)=0, \text { for }-d \leqslant x \leqslant d ; \\
& \frac{\partial B}{\partial x}=\text { finite, for all } t ; \text { and } \\
& B(d, t)=B^{\prime}, \text { for } t \geqslant 0 .
\end{aligned}
$$

Using finite Fourier transforms this problem can be solved analytically. A similar problem has been dealt with in a previous paper; ${ }^{18}$ in this paper only the solution for the electrical field at the surface of the sample (eg the induced voltage) will be given:

$$
E(t)=\frac{8}{\pi^{2}} \frac{B^{\prime} d}{\tau} \sum_{k} \exp \left(-k^{2} t / \tau\right),
$$

with $k=1,3,4 \ldots$ and $\tau=4 \mu_{0} \sigma_{\mathrm{n}} d^{2} / \pi^{2}$. For values of $t / \tau \gg 1$ only the first term of this series remains giving the exponential decay of Fig. 3 which is determined by the normal conductivity $\sigma_{\mathrm{n}}$ only. For smaller values of $t$, however, the model fails completely as expected. The experimental value of $\tau$ is $\tau=28 \mu \mathrm{s}$ which leads to a normal conductivity $\sigma_{\mathrm{n}}=9 \times 10^{8} \Omega^{-1} \mathrm{~m}^{-1}$. This value of $\tau$ has been observed in all experiments independent of flux jump field and sweep rate. This supports the simple assumptions made above because the solution of (9) starting from any initial condition yields a series expansion like (11), but with different coefficients.

In the experimental results a very high screening level (upto $4000 \mathrm{~A} \mathrm{~cm}^{-1}$ ) is observed which cannot be explained in terms of bulk pinning, but has to be attributed to surface currents. Therefore it is advantageous to introduce a model in which bulk currents are neglected and the sample is replaced by two current carrying surfaces. Now in the normal situation the following equation holds:

$$
\mu_{0} j_{\mathrm{s}}=B_{\mathrm{a}}-B_{\mathrm{i}}=\mu_{0} \sigma_{\mathrm{s}} d \frac{\mathrm{d} B_{\mathrm{i}}}{\mathrm{d} t}
$$
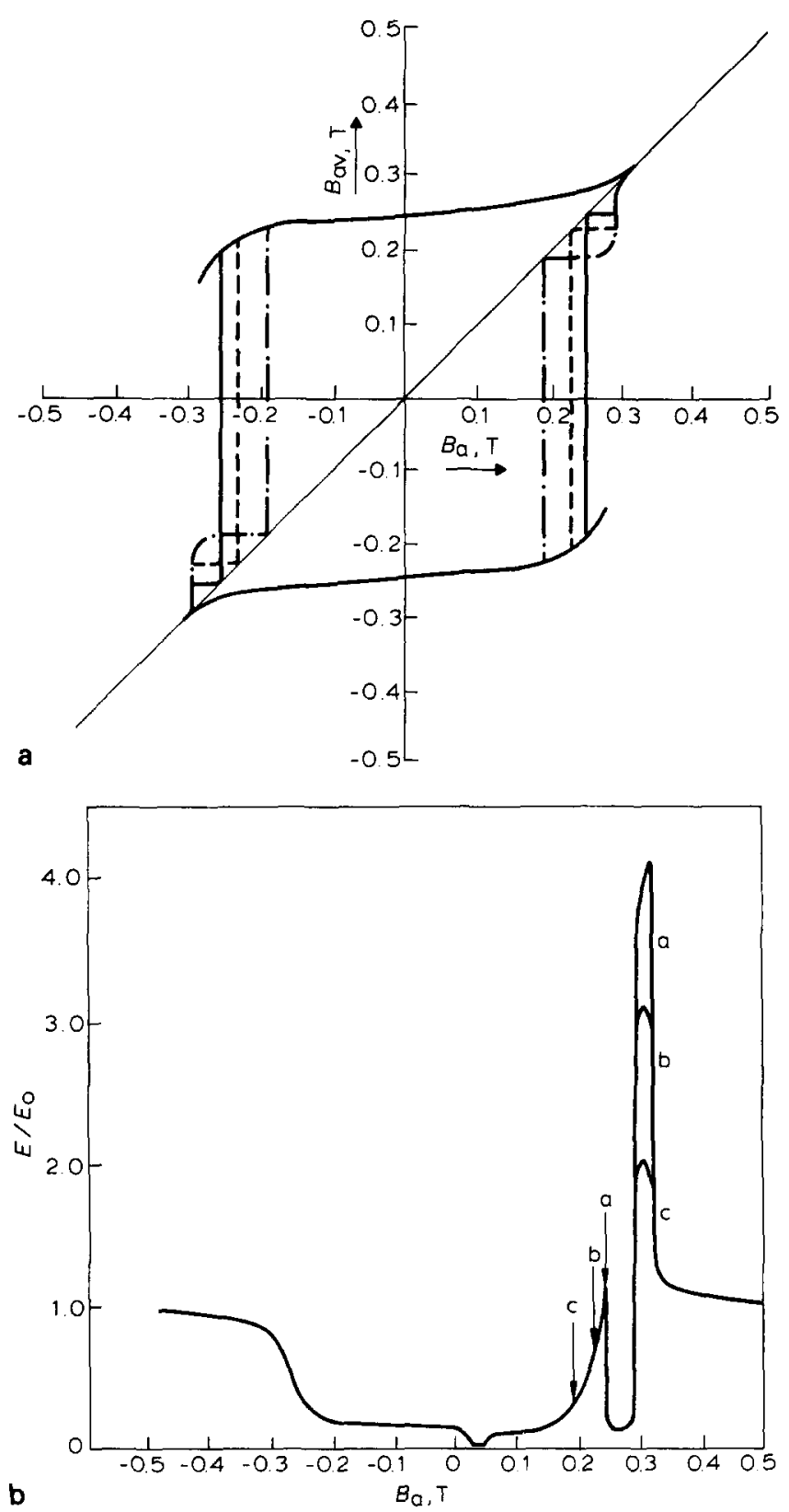

Fig. 6 a - Experimental hysteresis loops for a niobium single crystal for various values of $\mathrm{d} B_{\mathrm{a}} / \mathrm{d} t\left(b_{0}=500 \mathrm{mT}\right)$. The corresponding voltage peaks are given in Fig. $3 . \mathrm{b}-$ the corresponding induced voltage curves 


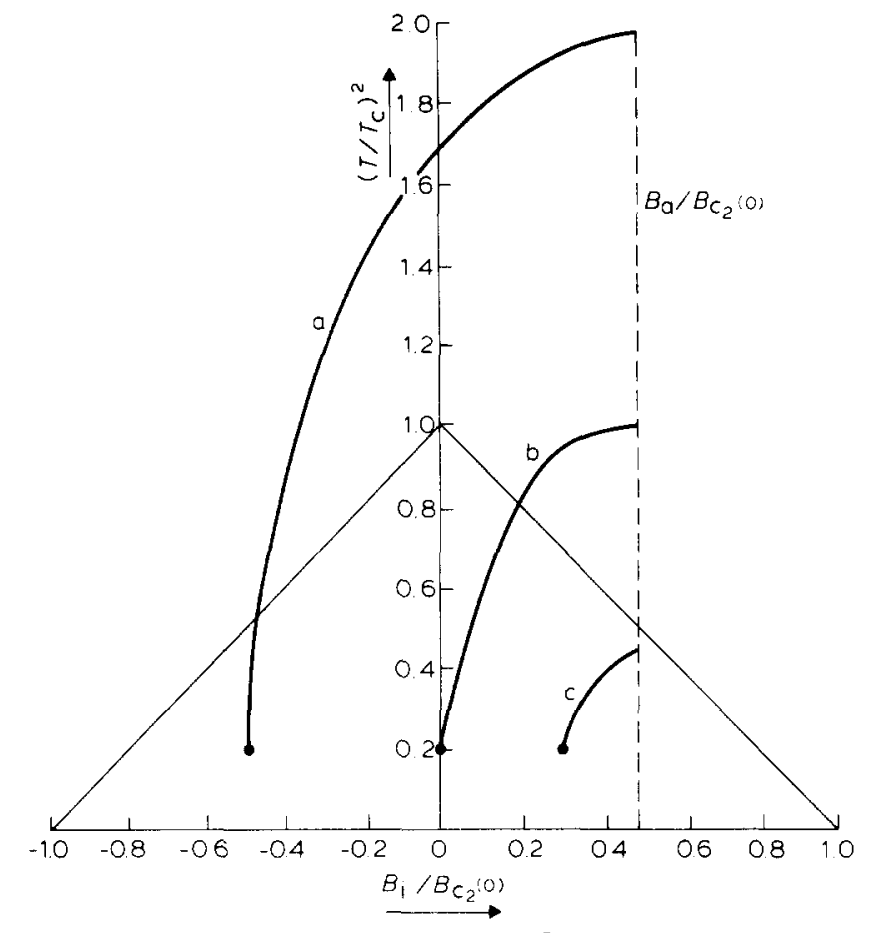

Fig. 7 Calculated trajectory in the $B_{1}-T^{2}$ plane of adiabatic flux jumps for three $\left(B_{\mathrm{i}}, B_{\mathrm{a}}\right)$-combinations: $a-b_{\mathrm{iO}}=B_{\mathrm{i} 0} / B_{\mathrm{c} 2}(0)$ $=-0.50, b_{\mathrm{aO}}=0.50 ; \mathrm{b}-b_{\mathrm{iO}}=0.0, b_{\mathrm{aO}}=0.50$ and $\mathrm{c}-b_{\mathrm{iO}}=0.30$, $b_{\mathrm{aO}}=0.50$

in which $j_{\mathrm{S}}$ is the surface current density, $\sigma_{\mathrm{s}}$ is the surface conductivity and $B_{\mathrm{i}}$ is the internal magnetic induction. The solution for the electric field at the surface reads:

$$
E(t)=\frac{B^{\prime} d}{\tau} \exp (-t / \tau)
$$

which equals (11) for large values of $t$ with $\tau=\mu_{0} \sigma_{\mathrm{s}} d$. For the solution of (12), the initial condition $B_{\mathrm{i}}(0)=0$ has been used.

The simplification of the slab to two current carrying surfaces also leads to a simplification of the heat diffusion equation, (2), since the dissipation is now restricted to the surface. In fact, the temperature distribution $T(x, t)$ now can be evaluated analytically, provided that the time dependence of $P(t)$ is known analytically too. In practical situations temperature profiles have been determined showing a rather fast and uniform temperature increase inside the sample. ${ }^{19}$ For strictly uniform temperatures (eg a very good heat conduction) the heat diffusion equation together with boundary condition $(8 \mathrm{c})$ and with $n=3$, reduces to:

$$
\frac{d T^{4}}{\mathrm{~d} t}=\frac{4\left(P_{\mathrm{s}}-Q_{0}\left(T-T_{0}\right)^{3}\right)}{C_{0} \cdot d}
$$

In (14) the usual temperature dependence $C=C_{0} T^{3}$ of the specific heat per unit volume is substituted. $P_{\mathrm{s}}$ is the heat dissipated in the surface layer given by:

$$
P_{\mathrm{s}}=j_{\mathrm{s}} \cdot E=\left(1 / \mu_{0}\right) \cdot\left(B_{\mathrm{a}}-B_{\mathrm{i}}\right) \cdot \mathrm{d} \frac{\mathrm{d} B_{\mathrm{i}}}{\mathrm{d} t}
$$

If the heat transfer to the surrounding helium may be neglected $\left(Q_{0}=0\right)$, a combination of (14) and (15) leads to:

$$
\frac{\mathrm{d} T^{4}}{\mathrm{~d} t}=\frac{4}{\mu_{0} C_{0}}\left(B_{\mathrm{a}}-B_{\mathrm{i}}\right) \frac{\mathrm{d} B_{\mathrm{i}}}{\mathrm{d} t}
$$

In a linearly increasing field, $B_{\mathrm{a}}=B_{\mathrm{a}}+\alpha t$ this can be integrated:

$$
\begin{aligned}
T^{4}-T_{0}^{4}= & \frac{2}{\mu_{0} C_{0}}\left\{2 B_{\mathrm{a} 0}\left(B_{\mathrm{i}}-B_{\mathrm{i} 0}\right)-B_{\mathrm{i}}^{2}+B_{\mathrm{i} 0}^{2}+2\right. \\
& \left.\int_{0}^{t} \alpha t^{\prime} \frac{\mathrm{d} B_{\mathrm{i}}}{\mathrm{d} t^{\prime}} \mathrm{d} t^{\prime}\right\}
\end{aligned}
$$

It is assumed here that before the jump the sample is at the same temperature as the surrounding helium; $B_{\mathbf{i o}}$ indicates the initial value of $B_{\mathrm{i}}$. If the sweep rate $\alpha$ is sufficiently low, the variation of $B_{\mathrm{a}}$ during the jump process may be neglected and (17) reduces to:

$$
T^{4}-T_{0}^{4}=\frac{2}{\mu_{0} C_{0}}\left(B_{\mathrm{i}}-B_{\mathrm{i} 0}\right)\left(2 B_{\mathrm{a} 0}-B_{\mathrm{i}}-B_{\mathrm{i} 0}\right)
$$

So in the adiabatic case, the temperature is a function of $B_{\mathrm{i}}$ only and independent of the thickness of the slab and of the form of the $j_{\mathrm{s}}\left(B_{\mathrm{i}}, E, T\right)$-relation. Fig. 7 shows the elliptic dependence of $T^{2}$ on $B_{1}$ for three sets of $\left(B_{\mathrm{i} 0}, B_{\mathrm{a} 0}\right)$ values in increasing external field. The maximum temperature $T_{\max }$ is reached for $B_{\mathrm{i}}=B_{\mathrm{a} 0}$ when the process ends:

$$
T_{\max }^{4}=T_{0}^{4}+\frac{2}{\mu_{0} C_{0}}\left(B_{\mathrm{a} 0}-B_{\mathrm{i} 0}\right)^{2}
$$

The curves in Fig. 7 reveal some interesting features. First, the final state can be inside the superconducting region (see curve c); in this case only a partical jump has occurred. This situation differs from the two other curves, where the final state is outside the superconducting region. In these two cases a distinction has to be made depending on the way the normal region is entered. In the case where much negative flux is frozen in, the bulk can become normal while there is still a large surface current. This is due to the fast temperature rise (see curve a). In this case $\left|B_{\mathrm{a}}\right|<B_{\mathrm{c} 2}<\left|B_{\mathrm{i}}\right|$ during some time interval. A similar behaviour does not occur in curve $b$; we will come back to this point later.

Although the $B_{\mathbf{i}}-T^{2}$ path is independent of the $j_{\mathrm{s}}\left(B_{\mathbf{i}}, E\right.$, $T$ )-relation, the latter plays an important role in describing the time dependence of all variables. For mathematical convenience a linear dependence of $j_{\mathrm{c}}$ on $B_{\mathrm{i}}$ was chosen. Analogous to bulk critical currents, it was assumed that a maximum critical surface current exists which amounts to:

$$
\mu_{0} j_{\mathrm{s}}=\beta\left(B_{\mathrm{c} 2}(T)-B_{\mathrm{i}}\right), \text { for }\left|B_{\mathrm{i}}\right|<B_{\mathrm{c} 2}(T)
$$

An isothermal hysteresis loop of a sample obeying (20) is shown in Fig. 8. As a consequence, a sample with internal flux density $B_{\mathbf{i} 0}$ will not show any flux penetration until the external field is increased to:

$$
B_{\mathrm{a}}=\beta B_{\mathrm{c} 2}(T)+(1-\beta) B_{\mathrm{i} 0}
$$

It can be seen from (21) that the factor $\beta$, ranging from 0 to 1 determines the external field that can be screened by a virgin sample. In fact the only two conditions that should be imposed on such a $j_{\mathrm{s}}\left(B_{\mathrm{i}}, E, T\right)$-relation are its ability to prevent flux penetration in some $B_{\mathrm{a}}$-range for any $B_{\mathrm{i}}$ value and a smooth vanishing of $j_{\mathrm{s}}$ when $B_{\mathrm{i}}$ approaches $B_{\mathrm{c} 2}(T)$.

During flux penetration over critical currents will also occur; in this case the total current can be written:

$$
j=p\left(B_{\mathrm{i}}, B_{\mathrm{a}}\right) \cdot j_{\mathrm{s}}+q\left(B_{\mathrm{i}}, B_{\mathrm{a}}\right) \sigma_{\mathrm{s}} E
$$


The function $q$ may be used to take into account the $B-$ dependence of the flux flow conductivity. In the following, however, $q$ is taken to be 1 for simplicity. The function $p\left(B_{\mathrm{i}}, B_{\mathrm{a}}\right)$ is introduced to allow a smooth variation of the supercurrents in the case $B_{\mathrm{i}}$ or $B_{\mathrm{a}}$ or both exceed $B_{\mathrm{c} 2}$, while there is still a large supercurrent at the surface (see Fig. 7: curve a). In Table 1 the quite arbitrary values of $p$, chosen for our calculations, are listed. The regions in this table refer to those in Fig. 8. These $p$-values provide a smooth variation both with $B_{\mathrm{i}}$ and $B_{\mathrm{a}}$; as a consequence the total current and the calculated induced voltage will also vary continuously in accordance with the experimental observations. The values $p=0$ and $p=1$ describe the normal and the completely superconducting state respectively.

Combination of Ampère's law $\mu_{0} j=B_{\mathrm{a}}-B_{\mathrm{i}}$, Faraday's law $E=\mathrm{d} . \mathrm{d} B_{\mathrm{i}} / \mathrm{d} t$ and (22) leads to the differential equation governing the time dependence of $B_{\mathrm{i}}$ :

$$
B_{\mathrm{a}}-B_{\mathrm{i}}=\mu_{0} p \cdot \beta\left[B_{\mathrm{c} 2}(T)-B_{\mathrm{i}}\right]+\mu_{0} \sigma_{\mathrm{s}} \mathrm{d} \frac{\mathrm{d} B_{\mathrm{i}}}{\mathrm{d} t}
$$

So in this model the flux jump process is completely described by (14), (23) and (15) which replace (2), (3) and (7) of the general description. The trajectory which the pro-

Table 1. The function $p\left(B_{1}, B_{\mathrm{a}}\right)$ for various regions in the $B_{i}-B_{\text {a }}$ plane

\begin{tabular}{|c|c|c|}
\hline 1 & Normal region & $p=0$ \\
\hline$\|$ & $\begin{array}{l}\text { Full superconducting } \\
\text { region }\end{array}$ & $\rho=\left(B_{\mathrm{a}}-B_{\mathrm{i}}\right) / \beta\left(B_{\mathrm{c} 2}-B_{\mathrm{i}}\right)$ \\
\hline III & Flux flow state & $p=1$ \\
\hline IV & $B_{\mathrm{a}}>B_{\mathrm{c} 2}$ & $p=\left(B_{\mathrm{c} 2}-B_{\mathrm{i}}\right) /\left(B_{\mathrm{a}}-B_{\mathrm{i}}\right)$ \\
\hline $\mathrm{v}$ & $B_{\mathrm{a}}<B_{\mathrm{c} 2}, B_{\mathrm{i}}<-B_{\mathrm{c} 2}$ & $\begin{array}{l}p=4 B_{\mathrm{c} 2}^{2} /\left(B_{\mathrm{a}}-B_{\mathrm{i}}\right) \\
\left(B_{\mathrm{c} 2}-B_{\mathrm{i}}\right)\end{array}$ \\
\hline VI & $B_{\mathrm{i}}<B_{\mathrm{c} 2}$ & $\begin{array}{l}p=2 B_{\mathrm{c} 2}\left(B_{\mathrm{a}}+B_{\mathrm{c} 2}\right) \\
{\left[\left(B_{\mathrm{a}}-B_{\mathrm{i}}\right)\left(B_{\mathrm{c} 2}-B_{\mathrm{i}}\right)\right]^{-1}}\end{array}$ \\
\hline VII & $B_{\mathrm{a}}>B_{\mathrm{c} 2}, B_{\mathrm{i}}<-B_{\mathrm{c} 2}$ & $\begin{array}{l}p=\left(B_{\mathrm{a}}+B_{\mathrm{c} 2}\right)^{2} \\
{\left[\beta\left(B_{\mathrm{a}}-B_{\mathrm{i}}\right)\left(B_{\mathrm{c} 2}-B_{\mathrm{i}}\right)\right]^{-1}}\end{array}$ \\
\hline
\end{tabular}

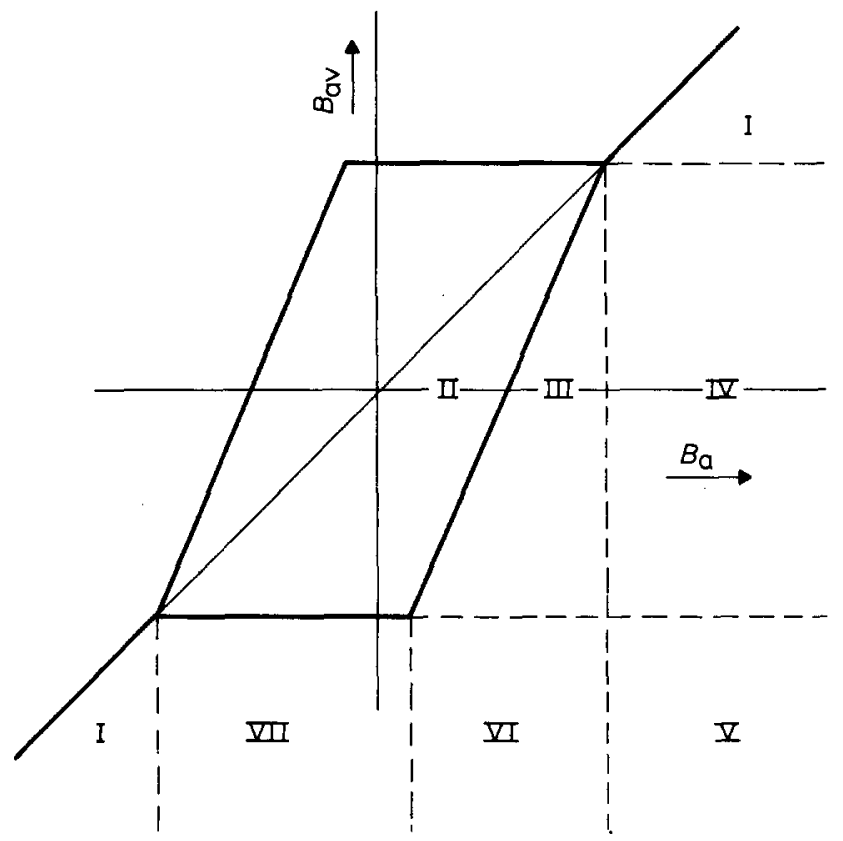

Fig. 8 The isothermal hysteresis loop used in the model calculations. The numbers refer to the various regions in Table 1 cess follows in the $B_{\mathrm{i}}-T^{2}$ plane is almost independent of $\alpha$, for small $\alpha$ and $Q_{0}=0$. The speed at which the process develops is closely related to the electrical conductivity $\sigma_{n}$ and found from (23). The results presented below are obtained by a simultaneous solution of (14), (23) and (15) in a large range of $\alpha$ - and $Q_{0}$ - values. In this way the validity of (17) can be verified for a large range of $\alpha$-values. Typical solutions for the induced voltage vs time curves for various values of $\alpha$ are shown in Fig. 9. The values of the parameters used have been listed in Table 2. The values of $\sigma_{\mathrm{s}}$ and $C_{0}$ have been chosen to fit the experiments. For $Q_{0}$ no data exist in the literature, but it has been assumed that the value for $\mathrm{NbT}^{20,21}$ is of the same order of magnitude. The value of $B_{\mathrm{i} 0}=-0.95$ $B_{\mathrm{c} 2}\left(T_{0}\right)$ has been chosen on the basis of the experimental results. By chosing the factor -0.95 it is expressed that the sample is still cooling down when the surface screening starts. Actually this factor can be calculated from a determination of the temperature variation during a complete cycle. These calculations have been omitted because the other approximations with respect to constants and relations are still rather crude.

The influence of the heat flow to the environment is shown in Fig. 10. In the adiabatic case $\left(Q_{0}=0\right)$ the final state is the normal state with $B_{\mathrm{i}}=B_{\mathrm{a}}$. In all other cases the sample will cool down to the starting temperature $T_{0}$ while the internal flux density is still smaller than $B_{\mathrm{a}}$.

For small values of $Q_{0}, B_{\mathrm{i}}$ is almost equal to $B_{\mathrm{a}}$ and one can speak of a complete flux jump; under very good cooling conditions an incomplete flux jump occurs. The path in the $B_{i}-T^{2}$ plane turned out to be rather independent of $\alpha$ in these cases. The corresponding induced voltage

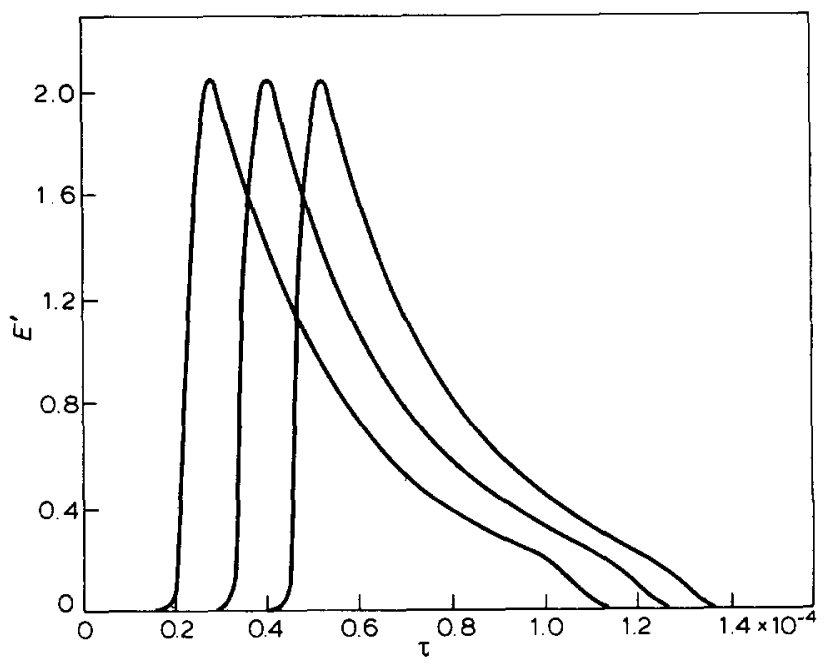

Fig. 9 Calculated voltage peaks for various values of $\alpha$. Along the ordinate the dimensionless voltage $E^{\prime}=E .\left(\mu_{0} \sigma_{s} / B_{\mathrm{c} 2}\right)$ is given. The dimensionless time $\tau$ equals $\tau=t / \mu_{0} \sigma_{s} d$

Table 2. Values of the parameters used in the calculations

\begin{tabular}{ll}
\hline$d$ & $2 \times 10^{-4} \mathrm{~m}$ \\
$T_{c}$ & $9.2 \mathrm{~K}$ \\
$T_{0}$ & $4.2 \mathrm{~K}$ \\
$B_{\mathrm{c2}}(0)$ & $0.4 \mathrm{~T}$ \\
$\beta$ & 0.6 \\
$\sigma_{\mathrm{s}}$ & $1.2 \times 10^{5} \Omega^{-1}$ \\
$C_{0}$ & $9.3 \mathrm{Jm}^{-3} \mathrm{~K}^{-4}$ \\
\hline
\end{tabular}




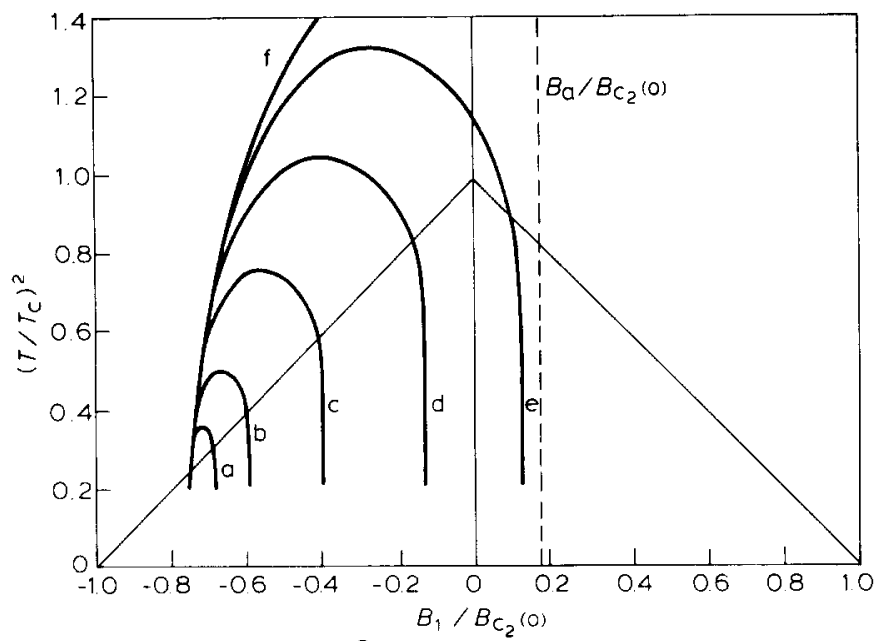

Fig. 10 Calculated $B_{i}-T^{2}$ curves for various conditions of heat transfer to the environment: $a-Q_{0_{3}}=6 \times 10^{4} \mathrm{Wm}^{-2} \mathrm{~K}^{-3}$; $\mathrm{b}-Q_{0}=2 \times 10^{4} ; \mathrm{c}-Q_{0}=6 \times 10^{3} ; \mathrm{d}-Q_{0}=2 \times 10^{3}$. e $-Q_{0}=6 \times 10^{2} ; f-$ the adiabatic curve $Q_{0}=0$

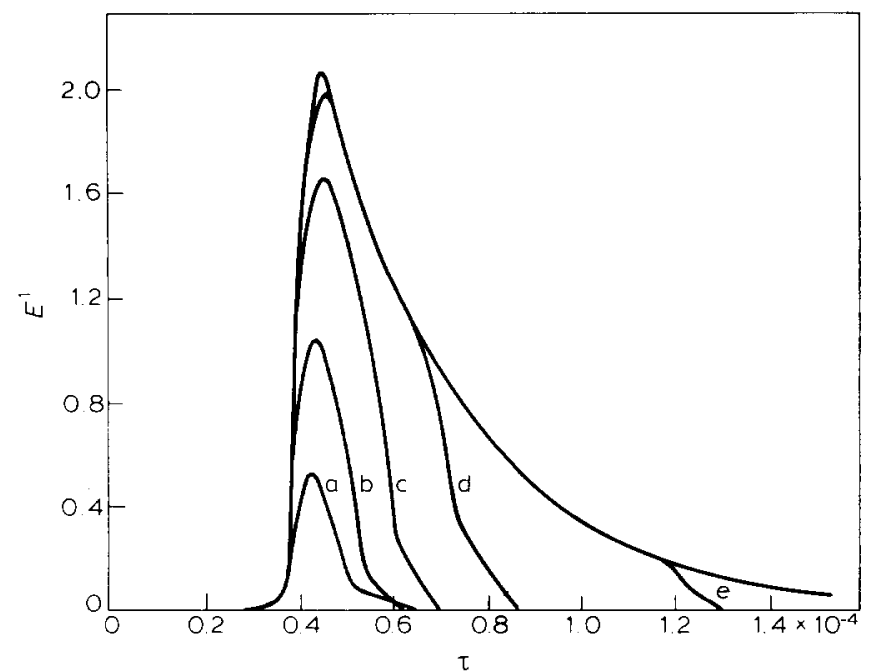

Fig. 11 Calculated voltage peaks for various values of $O_{0}$ (see Fig. 10)

curves, shown in Fig. 11 also show this characteristic. Here the $\alpha$-dependence only shows up by a time shift proportional to $\ln \alpha$. This latter point can be deduced from analytical arguments as well, but this is omitted here. The time shift increases with increasing $\alpha$ which contradicts the observed dependence. A possible explanation might be that with increasing frequency also, the cool down periods are shortened which results in lower jump fields.

\section{Conclusions}

The induced voltage from a niobium single crystal, exhibiting flux jumps has been investigated in a wide range of the field sweep rate $\alpha$ and compared with numerical model calculations. Because the general set of equations, (2), (3) and (7), which describe the flux jump process cannot yet be solved a much simpler model is introduced. In this model the supercurrents are restricted to the surface of the superconducting slab and a homogeneous temperature distribution inside the sample has been assumed. Good agreement between experimental results and computer calculations has been obtained (see Fig. 12). Especially the remarkable insensitivity of the shape of the observed induction peaks with varying sweep rate and amplitude can be explained by the model. It has been shown that the main parameter for describing the time dependence of the flux jump is the flux flow or

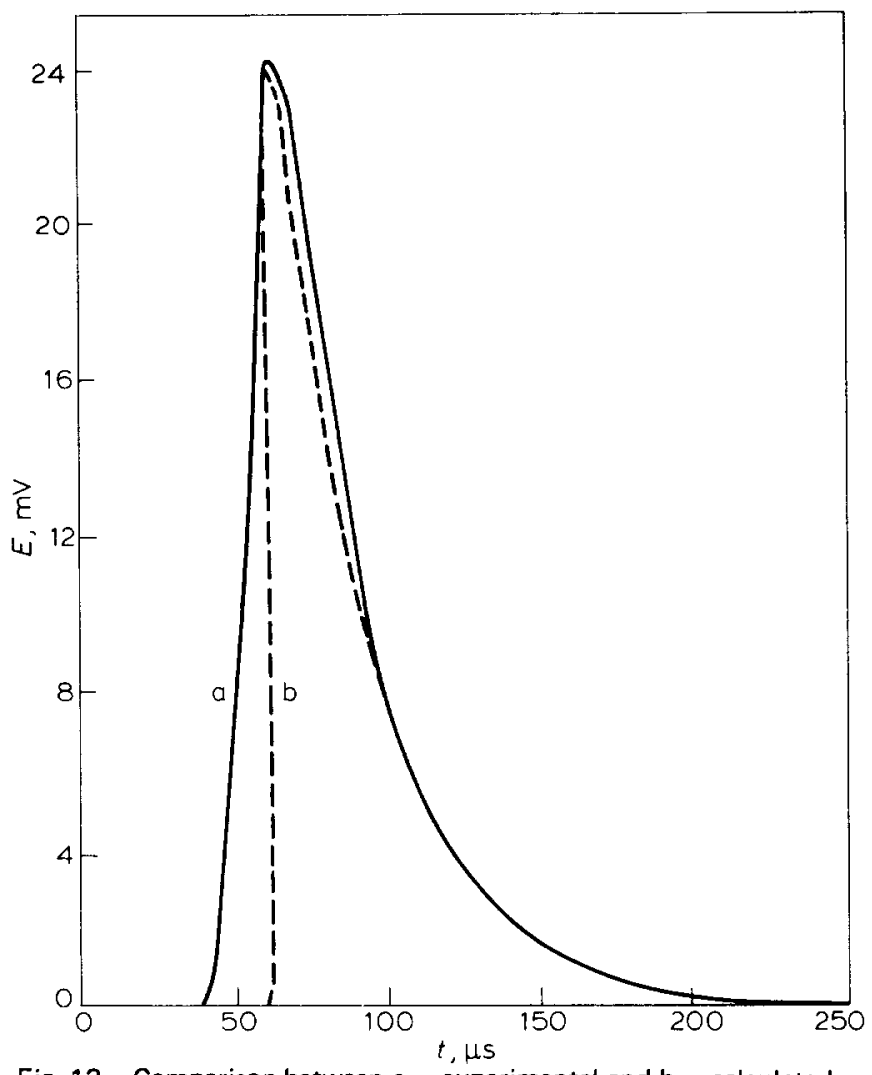

Fig. 12 Comparison between a - experimental and b - calculated voltage peak. The experimental curve has been measured with an amplitude $b_{0}=450 \mathrm{mT} ; \mathrm{d} B_{\mathrm{a}} / \mathrm{d} t=88 \mathrm{mT} \mathrm{s}^{-1}$. The flux jump field is $B_{\mathrm{fj}}=222 \mathrm{mT}$. The adiabatic curve has been calculated with the parameters given in Table 2

normal conductivity. Apart from a good agreement between experimental and numerical results at most points, a deviation has been observed with respect to the dependence of the jump field on the sweep rate. The reason for the very strong surface screening in this niobium single crystal is not yet understood.

\section{References}

\section{Hancox, R., Phys Lett 16 (1965) 208}

Swartz, P.S., Bean, C.P., J Appl Phys 39 (1968) 4991

Wipf, S.L., Phys Rev 161 (1967) 404

Melville, P.H., Adv Phys 21 (1972) 647

5 Takeo, M., Irie, F. Matsushita, T., Klipping, G., Lüders, K., Ruppert, U., preprint

6 Bean, C.P., Livingston, J.D., Phys Rev Lett 12 (1964) 14

7 de Gennes, P.G., Superconductivity of metals and alloys, Benjamin, New York (1966) 3, 76

8 Kim, S., Howard, R.E., Beasley, M.R., J Appl Phys 49 (1978) 730

9 Chikaba, J., Cryogenics 10 (1970) 306

10 Kaiho, K., Ohara, T., Koyama, K., Cryogenics 16 (1976) 103

11 Takeo, M., Proc. ICEC 7 London 1978, (IPC S̄cience and Technology Press) 673

12 Yamafuji, K., Takeo, M., Chikaba, J., Yano, N., Irie, F., J Phys Soc Japan 26 (1969) 315 and 30 (1971) 697

13 Brussière, J.F., LeBlanc, M.A.R., J Appl Phys 46 (1975) 406

14 Darby, M.I., Morton, N., J Compt Phys 13 (1973) 35

15 van de Klundert, L.J.M., Gijsbertse, E.A., van de Braak, H.P., Physica 94B (1978) 41

16 Brussière, J.F., IEEE Mag 13 (1977) 431

17 Gijsbertse, E.A., Caspari, M., van de Klundert, L.J.M., 21 5 (1981) 299

18 van de Klundert, L.J.M., van de Braak, H.P., Gijsbertse, E.A., Physica 90B (1977) 237

19 Boyer, L., Fournet, G., Mailfert, A., Noel, J.L., Rev Phys Appl 6 (1971) 501

20 Lyon, D.N., Advances on Cryogenic Engineering 10 (1965) 371

21 Efferson, K.R., J Appl Phys 40 (1969) 1995 